\title{
Henry Stapp Vs. David Bohm on Mind, Matter, and Quantum Mechanics
}

\author{
Paavo Pylkkänen ${ }^{1,2}$ (D) \\ Received: 30 January 2019 / Accepted: 21 March 2019 / Published online: 7 April 2019 \\ (C) The Author(s) 2019
}

\begin{abstract}
This paper briefly discusses some of David Bohm's views on mind and matter and suggests that they allow for a stronger possibility for conscious free will to influence quantum dynamics than Henry Stapp's approach.
\end{abstract}

Keywords Consciousness - Collapse of the wave function · Free will $\cdot$ Mental causation $\cdot$ Active information $\cdot$ Bohm theory $\cdot$ Stapp

\section{Introduction}

While Henry Stapp and David Bohm interacted constructively in various contexts (see, e.g., Griffin 1986), Stapp tends to be rather dismissive of Bohm's attempts to discuss the relationship of mind and matter in the context of quantum mechanics (see, e.g., Stapp 2007: 62; 132). Stapp, of course, acknowledges the value of Bohm's 1952 "causal" or "pilot wave" interpretation of QM, and Bohm and Hiley's later development of it under the label "ontological interpretation." He has also considered Bohm's more general implicate order approach and even made contributions that are useful for it (see Bohm 1986: 188; Stapp 1986). However, Stapp is critical of Bohm's proposal that the way to bring in mind to Bohm and Hiley's ontological interpretation is to extend the quantum ontology by adding higher level fields. In this paper, we briefly present some of Bohm's ideas on mind and matter. We then suggest that the advantage of Bohm's mind-matter scheme is that it allows for a stronger possibility for conscious free will to influence quantum dynamics than Stapp's approach, in which human "free choice" merely sets the stage for the entry of the statistical choice "nature" makes, obeying the Born rule.

Paavo Pylkkänen

paavo.pylkkanen@helsinki.fi

1 University of Helsinki, Helsinki, Finland

2 University of Skövde, Skövde, Sweden

\section{Bohm's Early Ideas on the Analogies between Quantum Processes and Thought}

David Bohm made many suggestions about how quantum theory might be relevant to understanding the mind and its relationship to matter. Already in his 1951 textbook Quantum Theory, he proposed that there are striking analogies between quantum processes and thought. For example, a kind of uncertainty principle applies for the thought process in the sense that an attempt to define the content of a stream of thought precisely will make the "direction" of the stream of thought indeterminate (see Bohm 1951: 169-172 and Pylkkänen 2014, 2015). He even suggested (inspired by Niels Bohr's writings) that these analogies could be explained if there were certain points controlling brain mechanisms so sensitive and delicately balanced that they must be described in an essentially quantum-mechanical way (he speculated that such points could exist at certain types of nerve junctions). In this way, Bohm anticipated the various "quantum mind" hypotheses that have appeared during recent decades.

\section{Bohm's 1952 Theory}

One of the main aims of Bohm's 1951 book Quantum theory was to explicate the physical meaning of the "orthodox" or Copenhagen interpretation (as it turned out, Bohm's view in the book is close to Wolfgang Pauli's view). After writing the book, Bohm was still not satisfied with quantum theory, feeling that something essential was missing, namely, a notion of "... an actual movement or activity by which one physical 
state could pass over into another one" (Bohm 1987). Discussions with Einstein in Princeton further encouraged him to look for a deterministic extension of quantum theory. In 1952, he published a "suggested interpretation of quantum theory in terms of hidden variables." He had independently discovered and improved the "pilot wave" theory which de Broglie had presented in the Solvay conference in 1927, but which had been dismissed at the time. In the Bohm theory, the electron is a particle with a well-defined position and momentum, guided by a new type field that is described by the wave function. The field gives rise to a quantum potential

$Q=-\frac{\hbar^{2}}{2 m} \frac{\nabla^{2} R}{R}$

where $R$ is the absolute value of the amplitude of the wave function $\Psi$. In the Bohm theory, the wave function $\Psi$ thus not only can be used to calculate probabilities, it also describes an objectively existing field that influences the particle through the quantum potential.

\section{Bohm's 1952 Theory and the Mind-Matter Relation}

A popular suggestion about the relationship between consciousness and quantum mechanics has to do with the role of the consciousness of the observer or experimenter in measurements (for example, such a role is crucial in Henry Stapp's (2007) view). Bohm's 1952 theory seems to eliminate any need for the consciousness of the observer in quantum dynamics or measurement-like interactions. Yet there is a sense in which the Bohm theory, too, opens up a new way for understanding the mind-matter relationship.

When Bohm reflected on the mathematical form of the quantum potential $Q$ in the late 1970s, he noticed that $Q$ only depends on the second spatial derivative of the amplitude $R$, i.e., on the way $R$ changes. This means that $Q$ depends only on the form or shape of the quantum field. This form typically reflects the form of the environment (e.g., whether one or two slits are open in a two-slit experiment). This suggests that the particle is not being pushed and pulled mechanically by the quantum field, but rather that the particle is able to respond to the form of the field, or is literally IN-FORMED actively by the information contained in the field. Note that this is information for the electron, not information for us. Thus, Bohm called this type of information active information. There exists potentially active information everywhere where the quantum potential is non-zero, while the information is actually active where the particle is (see Bohm and Hiley 1993). Another example of such objective and active information is the way the DNA molecule operates. It is the form of the DNA molecule which informs or guides protein construction.
How is this notion of active information relevant to the mind-matter relationship? Bohm noted that what is typical of mental phenomena is activity of form (as opposed to activity of substance). When we are reading a newspaper, we are abstracting the forms of the letters; we do not need to eat the paper. These forms are taken up by the nervous system and eventually give rise to an experience of meaning. The meaning, in turn, can be active (e.g., we may visit a certain store later on that day, if there was an interesting advertisement in the newspaper). Also, when we are reading a map, an information content builds up in the mind and guides our activities in the territory. Thus, there is at least an analogy between active information at the quantum level and active information in human subjective experience.

Bohm did not want to reduce the human mind and consciousness to the quantum level, but he also wanted to avoid dualism. He suggested that the quantum ontology can be extended to include higher level fields, each influencing and being influenced by levels below, and that the human mind could be a part of such a hierarchy of levels of information associated with certain neural processes (Bohm and Hiley (1993: 380-1; 386). In this way, say, when I move my hand, the information content in my thought could act down the hierarchy all the way to the level of the quantum field, which latter could then control particles (e.g., in synapses or some other relevant "quantum sites" in the brain). Such effects could then be amplified to control macroscopic neural processes.

Henry Stapp has been critical of postulating such an "infinite tower" of pilot waves to explain the mind: "Bohm certainly appreciated the need to deal more substantively with the problem of consciousness. He wrote a paper on the subject (Bohm 1990) which ended up associating consciousness with an infinite tower of pilot waves, each one piloting the wave below. But the great virtue of the original pilot-wave model namely the fact that it was simple and deterministic with cleanly specified solvable equations, became lost in this infinite tower" (Stapp 2007: 62). Bohm and Hiley acknowledged the difficulty and wrote: "...how can we understand this theory if the subtle levels are carried to infinity. Does the goal of comprehension constantly recede as we try to do this? We suggest that the appearance of such a recession is in essence just a feature of our language, which tends to give too much emphasis to the analytic side of our thought processes" (Bohm and Hiley 1993: 386-7). They further suggested that the infinity of levels is analogous to the poles of a magnet, which are a feature of linguistic and intellectual analysis, and have no independent existence outside of such analysis. At every part of a magnet there is a potential pair of north and south poles that overlap each other. But such potential poles are convenient abstractions that we introduce in order to think about the actuality, namely an unbroken magnetic field. 


\section{Stapp Vs. Bohm}

Henry Stapp has proposed a view which tries to reconcile the orthodox interpretation of quantum theory and our sense that we are free agents. This is an elegant approach but it is unsatisfactory in a crucial respect. Stapp emphasizes that there is a sense in which the "free choice" of the experimenter enters the quantum dynamics: "...prior to the appearance of an experimental outcome, a particular experiment needs to be set up. This 'setting up' partitions a continuum of quantum potentialities into a finite set of discrete possibilities." However, Stapp acknowledges that "...this 'free choice' merely sets the stage for the entry of the statistical choice between the ... alternatives whose relative statistical weights are specified by the quantum rules" (2007: 34). This means that in the end, it is "nature", not the free will of the human being that chooses what happens. And "orthodox quantum nature" obeys the Born rule, and in Stapp's approach there is thus a sense in which human action is the result of dice throwing. (However, one should acknowledge here that in his later work Stapp allows for a slight biasing or relaxation of the Born rule in order to accommodate the seeming backward-in-time causal effects reported by D.J. Bem and others (see Stapp 2017; this was pointed out to the author by one of the anonymous referees)).

Bohm's sketch of a mind-matter theory implies that the laws of quantum mechanics (and the Born rule) can be violated in those processes where higher order fields (including thoughts) act on the first-order quantum field. This means that human free will (in so far as it is possible for it to operate at the higher levels of information) can genuinely guide physical action. Difficult questions concerning, e.g., the possibility of non-local signaling certainly arise if one allows for violations of the Born rule (see, e.g., Valentini 2002; cf. Walleczek and Grössing 2016). However, if we are seeking a truly genuine causal efficacy of consciousness, those difficulties are worth facing in future research (see also Hiley and Pylkkänen 2005; Pylkkänen 2007 and 2017).

Funding Information Open access funding provided by University of Helsinki including Helsinki University Central Hospital.

\section{Compliance with Ethical Standards}

Conflict of Interest The author declares that he has no conflict of interest.

Open Access This article is distributed under the terms of the Creative Commons Attribution 4.0 International License (http:// creativecommons.org/licenses/by/4.0/), which permits unrestricted use, distribution, and reproduction in any medium, provided you give appropriate credit to the original author(s) and the source, provide a link to the Creative Commons license, and indicate if changes were made.

\section{References}

Bohm, D. (1951). Quantum theory. New York: Prentice-Hall [also New York: Dover, 1989].

Bohm, D. (1986). Time, the Implicate Order, and Pre-space. In Griffin ed. 1986. Physics and the Ultimate Significance of Time: Bohm, Prigogine, and Process Philosophy. Albany: State University ofNew York Press.

Bohm, D. (1987). Hidden variables and the implicate order. In B. J. Hiley \& F. D. Peat (Eds.), Quantum implications: Essays in honour of David Bohm. London: Routledge.

Bohm, D. (1990). A new theory of the relationship of mind and matter. Philosophical Psychology, 3(2), 271-286.

Bohm, D., \& Hiley, B. J. (1993). The Undivided Universe: An Ontological Interpretation of Quantum Theory. Lontoo: Routledge.

Griffin, D. (Ed.). (1986). Physics and the Ultimate Significance of Time: Bohm, Prigogine, and Process Philosophy. Albany: State University of New York Press.

Hiley, B. J., \& Pylkkänen, P. (2005). Can mind affect matter via active information? Mind and Matter, 3(2), 7-26 URL = http://www. mindmatter.de/resources/pdf/hileywww.pdf. Accessed January 15 2019.

Pylkkänen, P. (2007). Mind, Matter and the Implicate Order. Heidelberg and New York: Springer Frontiers Collection.

Pylkkänen, P. (2014). Can quantum analogies help us to understand the process of thought? Mind and Matter, 12/1, 61-92 (This is a modified version of Pylkkänen 2004). URL $=\mathrm{http}: / /$ www.mindmatter. de/resources/pdf/pylkkaenen_www.pdf.

Pylkkänen, P. (2015). Fundamental physics and the mind - is there a connection? In H. Atmanspacher, C. Bergomi, T. Filk, \& K. Kitto (Eds.), Quantum Interaction 2014: 8th International Conference, QI 2014, selected papers (pp. 3-11). Heidelberg: Springer. https://doi. org/10.1007/978-3-319-15931-7_1.

Pylkkänen, P. (2017). Is there room in quantum ontology for a genuine causal role of consciousness? In A. Khrennikov \& E. Haven (Eds.), The Palgrave handbook of quantum models in social science. Palgrave Macmillan.

Stapp, H. (1986). Einstein Time and Process Time. In Griffin ed. 1986. Physics and the Ultimate Significance of Time: Bohm, Prigogine, and Process Philosophy. Albany: State University of New York Press.

Stapp, H. (2007). Mindful Universe: Quantum Mechanics and the Participating Observer. Berlin and New York: Springer Frontiers Collection.

Stapp, H. (2017) Retrocausation in quantum mechanics and the effects of minds on the creation of physical reality, AIP Conference Proceedings 1841, 040001 (2017); https://doi.org/10.1063/1. 4982777.

Valentini, A. (2002). Signal-locality and subquantum information in deterministic hidden-variables theories. In T. Placek \& J. Butterfield (Eds.), Non-locality and modality (pp. 81-103). Dordrecht: Kluwer.

Walleczek, J., \& Grössing, G. (2016). Nonlocal quantum information transfer without superluminal signalling and communication. Foundations of Physics, 46(9), 1208-1228 https://rdcu.be/bi73o.

Publisher's Note Springer Nature remains neutral with regard to jurisdictional claims in published maps and institutional affiliations. 